\title{
Frequency of Fat Necrosis in Mammography and Sonography and Correlation with Pathology for Differentiation of Breast Cancer in Breast Cancer Research Center
}

DOI: $10.21859 /$ mci-supp-111

\section{Keywords:}

Necrosis

Mammography

Sonography

Breast Cancer

\author{
Toktam Beheshtian ${ }^{1, *}$, Asiie Olfatbakhsh ${ }^{1}$, Esmatosadat Hashemi ${ }^{1}$, \\ Fateme Sari ${ }^{1}$ \\ ${ }^{1}$ Breast Disease Department, Breast Cancer Research Center, Motamed Cancer \\ Institute, ACECR, Tehran, Iran \\ ${ }^{*}$ Corresponding author: Toktam Beheshtian, Breast Disease Department, Breast \\ Cancer Research Center, Motamed Cancer Institute, ACECR, Tehran, Iran. E-mail: \\ tbeheshtian@gmail.com
}

\begin{abstract}
Introduction: Fat necrosis is a low prevalence phenomenon that may be controversial for the radiologist and physicians to diagnose. The aim of this retrospective study was to evaluate the prevalence of the diagnosis of fat necrosis in mammography and ultrasound of patients to determine the imaging views of those patients who were operated.

Materials and Methods: By referring to the imaging center of Breast Cancer Research, all mammography, ultrasound and pathology results in a period of one year since January 2014 till Dec 2015 were evaluated. The data were entered and analyzed by SPSS software. Results: Overall 9512 breast ultrasound were performed in the same period, of which 103 had a diagnosis of fat necrosis (1.08\%). Also of 7116 mammogram, fat necrosis was found in 43 cases $(0.6 \%)$. The 196 cases of fat necrosis were diagnosed by imaging. The mean age of non-operated patients was 48 years and of operated ones was 42.6 years. The most common finding in the ultrasound of operated patients were heteroechoic mass + calcification (30.7\%). In the non-operated group, Hypoechoic mass + calcification was the most common feature. The most common mammographic findings in the operated group were calcification (20\%) and then distortion + calcification (13.7\%). In the other group, the most common mammographic findings were calcification (15.8\%) and Asymmetric density + calcification (15.8\%). In all patients, fat necrosis was reported in $74.3 \%$ of sonography reports and $45 \%$ of mammography reports. In the group who were not operated, 122 patients (83\%) had a history of previous surgery, but in operated cases $(31.4 \%)$ had a history of previous surgery.

Conclusions: These findings showed that views of fat necrosis are highly variable in mammography and ultrasound from BIRADS 2 to 4 . Among the imaging modalities ultrasound seems to be more sensitive than mammography in detecting these lesions, but based on the literature, mammography is more specific.
\end{abstract}

